\title{
Tuning a Two-Chamber Muffler for Wide-Band Transmission Loss
}

\author{
M. L. Munjal \\ Facility for Research in Technical Acoustics (FRITA), Department of Mechanical Engineering, Indian Institute of \\ Science, Bengaluru - 560 012, India.
}

(Received 12 October 2019; accepted 3 January 2020)

The design of a muffler with wide-band transmission loss (TL) is a basic requirement for automotive exhaust systems where the exhaust noise is spread over several harmonics of the engine firing frequency. This has led to the concept of a double-tuned expansion chamber. In the present paper, the concept of double-tuning is extended to the two-chamber mufflers. The author's classical Velocity Ratio Algorithm is used here to analyze and design such a muffler. The relevant two-row array is manipulated and simplified to arrive directly at the single most significant term out of the 81 non-dimensional terms constituting the relevant Velocity Ratio. Examination of this single term reveals acoustics of the raised wide-band domes of the TL spectrum of the double-tuned two-chamber (DT2C) muffler vis-a-vis that of an equivalent longer double-tuned single-chamber muffler. Except for a low-frequency trough, its TL curve covers the entire frequency range of interest for control of automotive exhaust noise. It is also shown that the corresponding side-inlet side-outlet (SISO) DT2C muffler configuration has an identically similar wide-band TL spectrum to that of the co-axial muffler configuration. Finally, some guidelines are laid out for designing a DT2C muffler.

\section{INTRODUCTION}

The transmission loss (TL) spectrum of a simple expansion chamber (SEC) muffler (Fig. 1(a)) is characterized by sharp periodic troughs touching zero $\mathrm{dB}$ (Fig. 1(c)) at frequencies given by:

$$
f_{n}=n \frac{c_{0}}{2 L}, n=1,2,3, \ldots \text {; }
$$

where $c_{0}$ is the speed of sound and $\mathrm{L}$ is the length of the chamber. The corresponding insertion loss (IL) spectrum also would have these troughs apart from those due to the low-frequency dip (system resonance) and the tail pipe resonances. These troughs result in very low overall IL due to the arithmetic of logarithmic addition. ${ }^{1}$ Fortunately, three-fourths of these periodic troughs can be tuned out by extending the inlet pipe (or exhaust pipe) and outlet pipe (or tail pipe) of the muffler into the expansion chamber by $L / 2$ and $L / 4$, respectively, (Fig. 1(b)), minus the end-corrections. ${ }^{2-4}$ The $L / 2-$ extension nullifies or tunes out all odd-numbered $(n=1,3,5,7, \ldots)$ troughs and the $L / 4$ extension tunes out the $n=2,6,10, \ldots$ troughs. This operation, called double-tuning, converts the simple expansion chamber (SEC) of Fig.1a into a double-tuned extended-tube chamber (DTETC) of Fig. 1(b). Consequently, the first four domes of the SEC-TL graph get replaced by a single overarching dome that, at its peak, stands about $12 \mathrm{~dB}$ above the SEC domes (see Fig. 1(c)). This can also be applied to the SISO chamber. ${ }^{5}$

However, at the extended inlet/outlet junctions, evanescent higher-order modes are produced, the effect of which on plane wave propagation is to introduce lumped inertance which can be accounted for through the so-called end-corrections - see, for example, Åbom, ${ }^{6}$ Peat, ${ }^{7}$ Selamet and $\mathrm{Ji}^{8-10}$ Torregrosa et al., ${ }^{11}$ Kang and $\mathrm{Ji}^{12}{ }^{12}$ Mimani and Munjal, ${ }^{13}$ and Gaonkar et al., ${ }^{14}$ among others.

The concept of tuning out the TL-troughs due to chamber resonances by appropriately sizing the extended inlet and outlet was also demonstrated by Selamet and $\mathrm{Ji}^{8}$ for singlechamber mufflers and Selamet et al. ${ }^{15}$ for the dual-chamber mufflers. The resultant lengths of the extended inlet and outlet

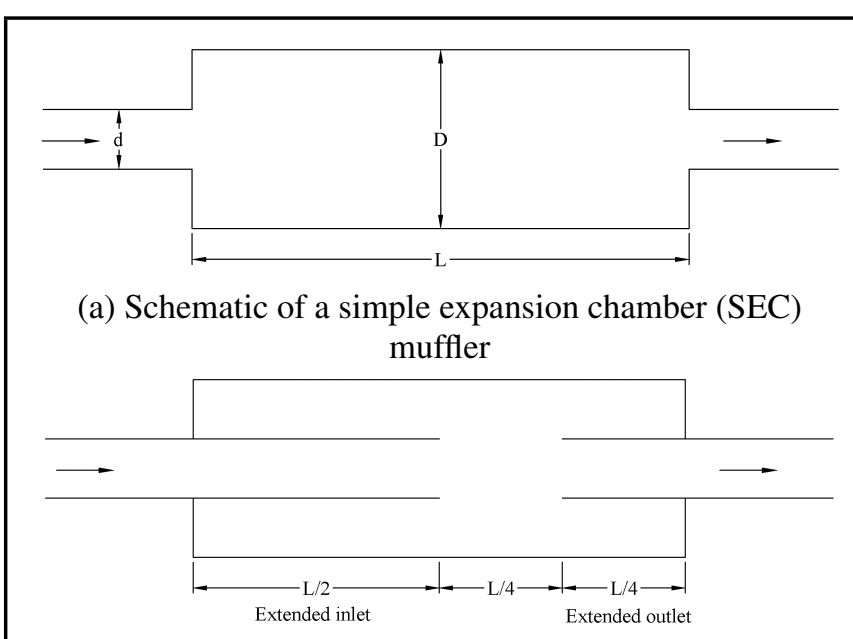

(b) Schematic of a double-tuned extended-tube chamber (DTETC)

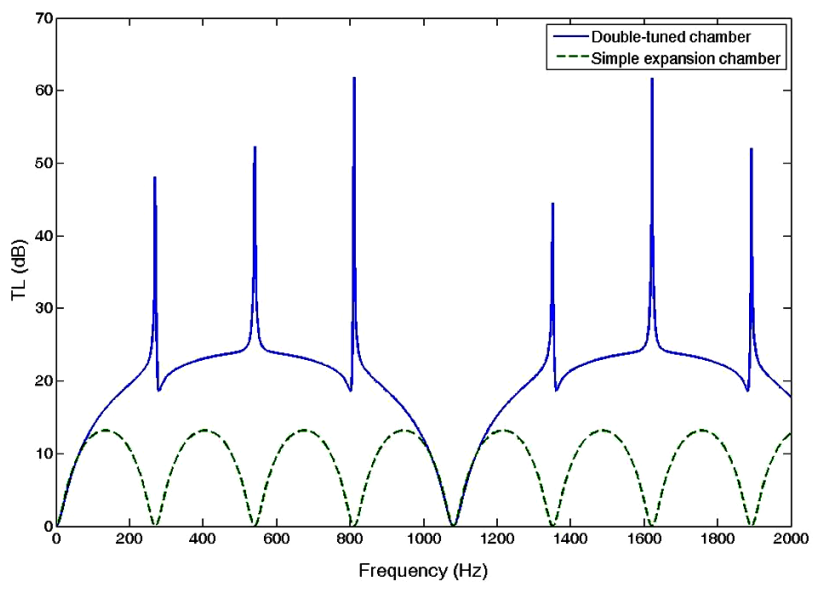

(c) Comparison of the TL spectra of the SEC and DTETC muffler

Figure 1. Concept of double-tuning of an expansion chamber 


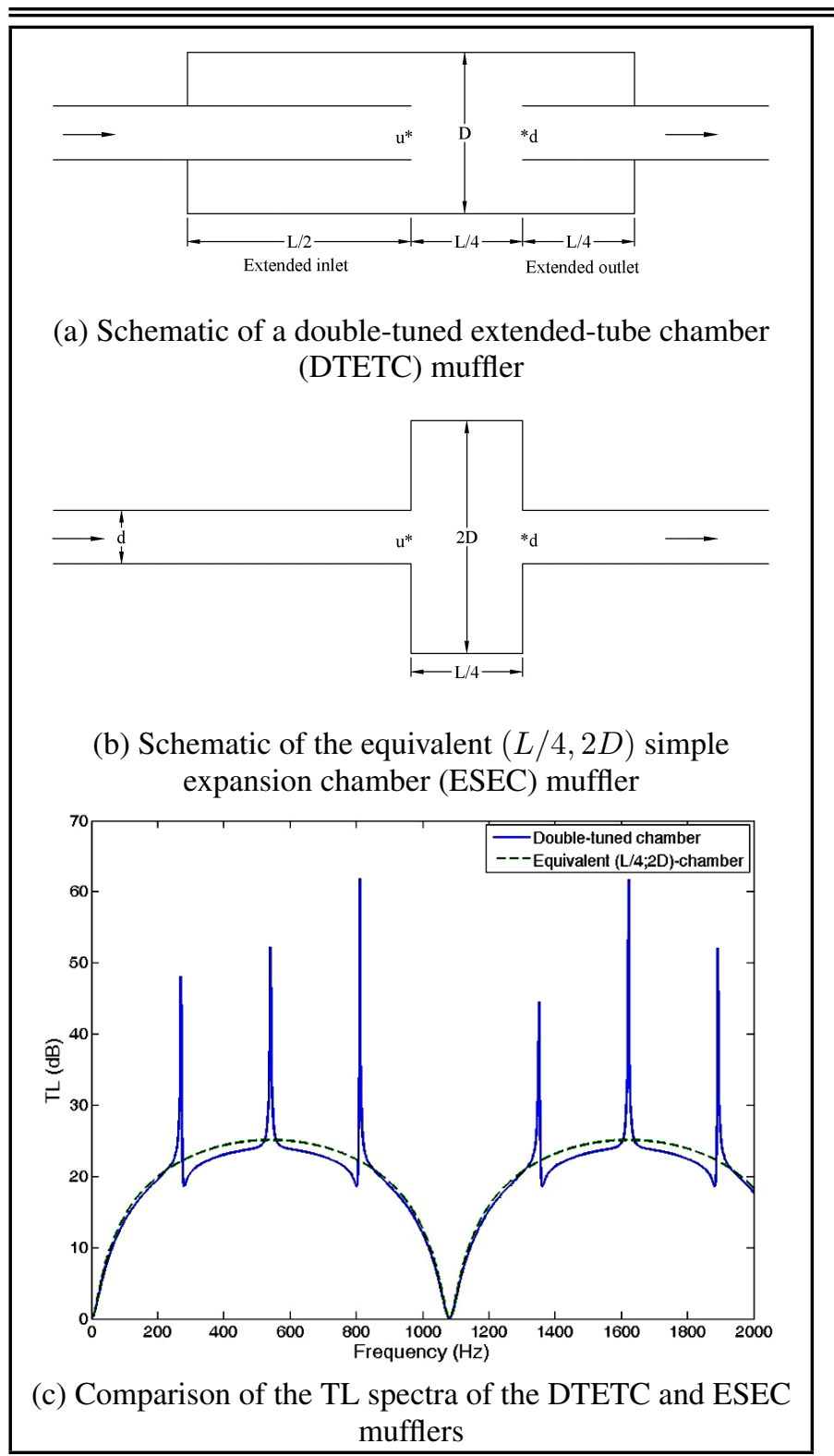

Figure 2. Illustration of Equivalence of the DTETC and ESEC mufflers

turn out to be $L / 2-\sigma$ and $L / 4-\sigma$, respectively, where $\sigma$ is the relevant end-correction. ${ }^{4}$

In this investigation, we extended this double-tuning concept to a two-chamber muffler within the same total length $L$ in order to analytically explore the possibility of obtaining a still wider and higher TL graph. This was accomplished here by means of the author's classical Velocity-Ratio $(V R)$ Algorithm, ${ }^{16,17}$ which has been found to be particularly useful in rational synthesis of one-dimensional acoustic filters or mufflers ${ }^{18}$ as well as multi-degree-freedom vibration isolators. ${ }^{19}$

Sharp resonance peaks were of no practical use. The muffler designer should concentrate on the underlying wide TL domes. Recently, Gaonkar and Munjal ${ }^{5}$ have shown analytically that the underlying wide TL domes can be replicated by replacing the double-tuned extended-tube chamber by a simple expansion chamber of length $L / 4$ and diameter $2 D$, as shown in Fig. 2. This equivalence comes in handy in analysis and development of the DT2C mufflers, as illustrated in the following sections.

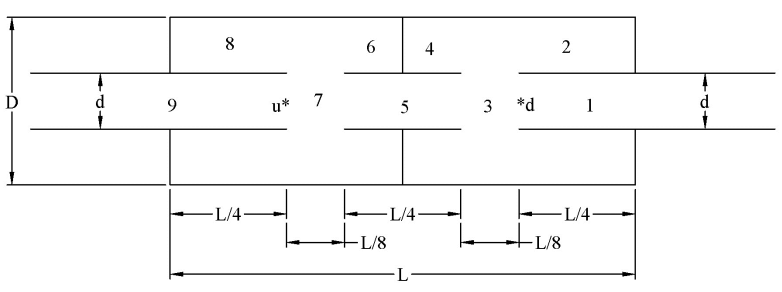

(a) Schematic of a double-tuned two-chamber (DT2C) muffler with total length $L$.

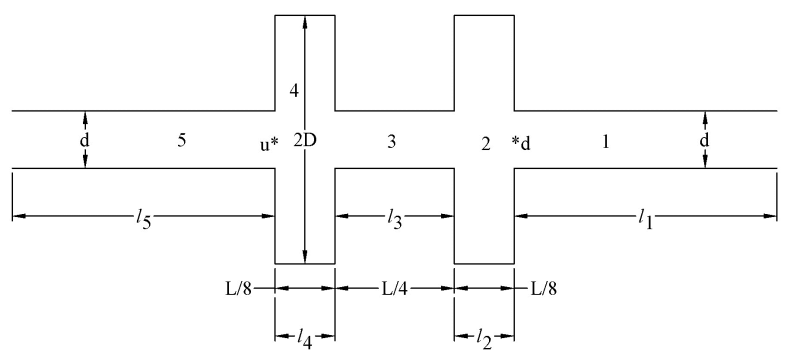

(b) Schematic of the equivalent $(L / 4,2 D)$ two-chamber muffler for configuration (a)

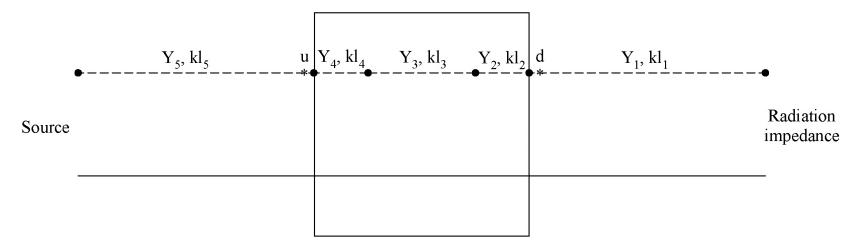

(c) Electrical analogous circuit for the muffler configuration (b)

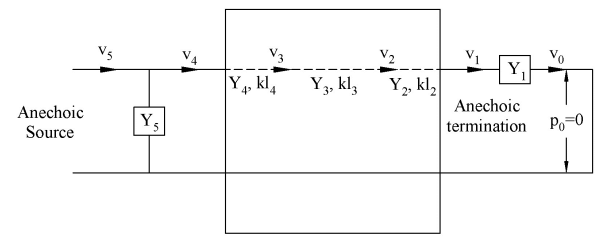

(d) Electrical analogous circuit (c) modified for anechoic source and load (termination)

Figure 3. Evaluation of TL of a DT2C muffler using the equivalent configuration and electroacoustic analogies

\section{ANALYSIS OF THE DT2C MUFFLER USING THE VR ALGORITHM}

Schematic of a DT2C muffler with the same overall length $L$ and its equivalent $(L / 4,2 D)$ configuration and electrical analogous circuit are shown in Fig. 3(a), Fig. 3(b) and Fig. 3(c), respectively. Transmission loss, by definition, presumes/requires anechoic source and load, as shown in Fig. 3(d). Transmission loss may now be evaluated from velocity ratio as follows. 5,16

$$
\begin{gathered}
V R_{5} \equiv\left(v_{5} / v_{0}\right)_{p_{0}=0} ; \\
T L=20 \log \left|\frac{v_{5} / v_{0}}{2}\right|_{p_{0}=0} ; \\
Y_{1}=Y_{3}=Y_{5}=Y_{0}=c_{0} /\left(\frac{\pi}{4} d^{2}\right), \\
Y_{2}=Y_{4}=c_{0} /\left(\frac{\pi}{4}(2 D)^{2}\right)=\frac{Y_{0}}{4 m}, m \equiv(D / d)^{2} .
\end{gathered}
$$

The velocity ratio $V R_{5}$, defined by Eq. (2), may be evaluated by means of the Author's VR-Algorithm ${ }^{17}$ without having to write and multiply out five transfer matrices as follows. ${ }^{1,17}$ 
Velocity ratio being non-dimensional would be an algebraic sum of $2^{4}=16$ non-dimensional terms corresponding to valid combinations of subscripts 1 to 5 (representing characteristic impedances $Y_{1}$ to $Y_{5}$ ) arranged in the two-row array: $:^{1,17,18}$

$$
\left[\begin{array}{lllll}
1 & 2 & 3 & 4 & \\
& 2 & 3 & 4 & 5
\end{array}\right]
$$

and sine or cosine of $k l_{2}, k l_{3}$ and $k l_{4}$ as per certain rules. ${ }^{1,17}$ As $Y_{2}=Y_{4}=m Y_{3}, m \gg 1$ the relatively more significant (higher-order) terms would be given by the valid combinations of the modified 2-row array: 1,17,18

$$
\left[\begin{array}{lllll}
1 & & 3 & & \\
& 2 & & 4 & 5
\end{array}\right]
$$

The most significant combination will be the one having both $Y_{2}$ and $Y_{4}$ in the denominator. Thus: ${ }^{1,17,18}$

$$
V R_{5} \sim-j \frac{Y_{1} Y_{3}}{Y_{2} Y_{4}} \sin \left(k l_{2}\right) \sin \left(k l_{3}\right) \sin \left(k l_{4}\right) .
$$

Substituting the lengths indicated in Fig. 3(b) and relative values of $Y$ in Eq. (4) yields:

$$
\left|V R_{5}\right| \sim\left(4 m^{2}\right) \sin ^{2}\left(\frac{k L}{8}\right) \sin \left(\frac{k L}{4}\right) ;
$$

By searching for the maxima and minima of $\sin ^{2} \theta \sin 2 \theta, \theta \equiv k L / 8, \quad$ it can be readily shown that Eq. (8) would peak at $\frac{k L}{8}=\frac{\pi}{3}$ and $\frac{2 \pi}{3}$ and drop in sharp trough inbetween at $\frac{k L}{8}=\frac{\pi}{2}$. Substituting $k=\omega / c_{0}=2 \pi f / 346, L=640 \mathrm{~mm}=0.64 \mathrm{~m}$, it can be seen that the TL peaks would occur at:

$$
f_{p} \simeq 720 \text { and } 1440 \mathrm{~Hz} ;
$$

and the intermediate trough at:

$$
f_{t} \simeq 1080 \mathrm{~Hz}
$$

As:

$$
20 \log \left(\sin ^{2} \frac{\pi}{3} \sin \frac{2 \pi}{3}\right)=60 \log (0.866)=-3.75 \simeq-4 d B
$$

and:

$$
m=(D / d)^{2}=(0.09 / 0.03)^{2}=9 ;
$$

the peak value of transmission loss would be given by:

$$
\begin{aligned}
T L_{\text {peak }} \simeq & 20 \log \frac{\left|V R_{5}\right|}{2}= \\
& 20 \log \frac{(4 \times 9)^{2}}{2}-3.75=62-6-4=52 \mathrm{~dB} .
\end{aligned}
$$

These deductions from the single most significant term are borne out by Fig. 4, which is drawn from the proper transfer matrix multiplication (i.e., by considering all 16 terms that constitute the velocity ratio)

Similar exercise using the VR-algorithm on the doubletuned single chamber muffler configuration of Fig. 2(a) and its equivalent $(L / 4,2 D)$ simple expansion chamber configuration of Fig. 2(b) yields:

$$
\begin{aligned}
& V R_{3} \sim(4 m) \sin (k L / 4) \text { and } \\
& \qquad T L_{\text {peak }} \simeq 20 \log \left|\frac{4 \times 9}{2}\left(\sin \frac{\pi}{2}\right)\right| \simeq 25 d B .
\end{aligned}
$$

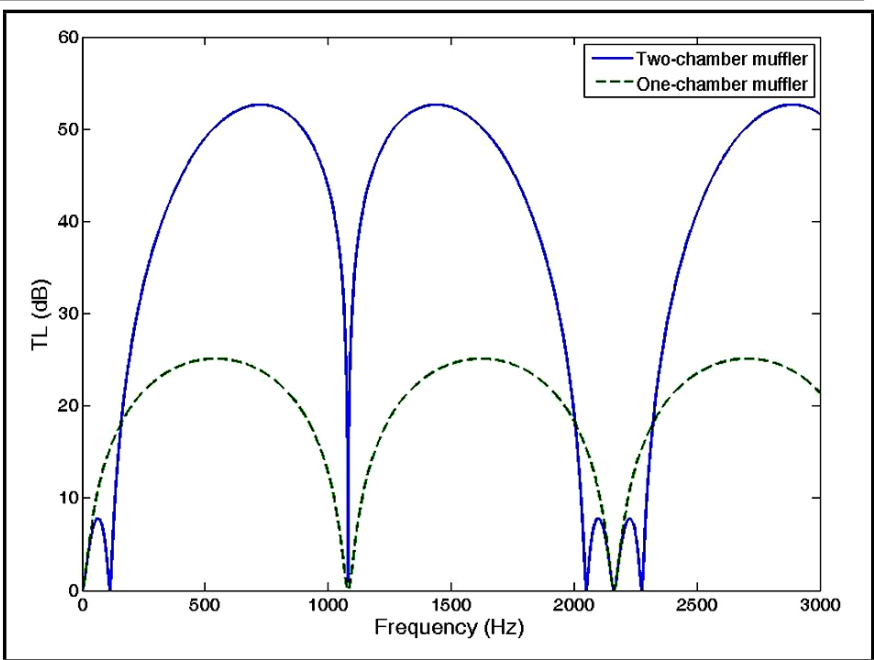

Figure 4. Effect of the number of double-tuned chambers within the same overall length, using the equivalent muffler configurations

This is also borne out by the dashed curve in Fig. 4.

This brings out the power of the author's algebraic algorithm not only for simplifying the analysis of a complex muffler but also for synthesizing an efficient muffler configuration.

Figure 4 compares the TL graphs of the double-tuned twochamber muffler and single-chamber muffler within the same overall length, $L=640 \mathrm{~mm}$. Other dimensions are $d=$ $30 \mathrm{~mm}, D=3 d=90 \mathrm{~mm}$. Obviously, the two-chamber muffler is better (rather, much better) than the single-chamber muffler in the frequency range $150 \mathrm{~Hz}$ to about $1950 \mathrm{~Hz}$. Of course, these values would change with the overall muffler length $L$, yet this frequency range would cover the entire frequency range of interest for a multi-cylinder automotive engine.

\section{SINGLE-TUNED COAXIAL MUFFLER}

TWO-CHAMBER

An alternative configuration of a tuned 2-chamber muffler where the intermediate pipe is extended half way into each of the two chambers but the exhaust pipe and tailpipe are not ${ }^{10}$ extended into the respective chambers, is shown in Fig. 5. This is one of the several configurations analyzed using classical plane-wave theory and validated experimentally by Davis et al. ${ }^{20}$ Dynamically, each of the two identical chambers of the muffler of Fig. 5(a) can be looked upon as singletuned or semi-tuned expansion chamber analysed recently by Gaonkar and Munjal. ${ }^{21}$ They showed that the base TL curve of the tuned chamber is similar to that of the corresponding SEC with effective length reduced to half $(L / 2)$ and area ratio $m=(D / d)^{2}$ increased to double (cf. the doubly-tuned chamber of Fig. 2 where the effective length is reduced to onefourth $(L / 4)$ and area ratio $m$ is increased to four fold). Thus, the basic TL domes can be captured by simplifying Fig. 5(a) to the equivalent $(L / 2, \sqrt{2} D)$ two-chamber muffler shown in Fig. 5(b). The electrical analogous circuit for the muffler configuration Fig. 5(b) would be identical to Fig. 3(c) except that $l_{2}=L / 4, l_{3}=L / 2$ and $l_{4}=L / 4$ (all double of the corresponding lengths in Fig. 3(b)). Fig. 3(d) would also apply to the configuration Fig. 5(b).

Now, applying the author's VR-algorithm and identifying the most significant term, Eq. (8) would get modified to:

$$
\left|V R_{5}\right| \sim(2 m)^{2} \sin ^{2}\left(\frac{k L}{4}\right) \sin \left(\frac{k L}{2}\right) .
$$




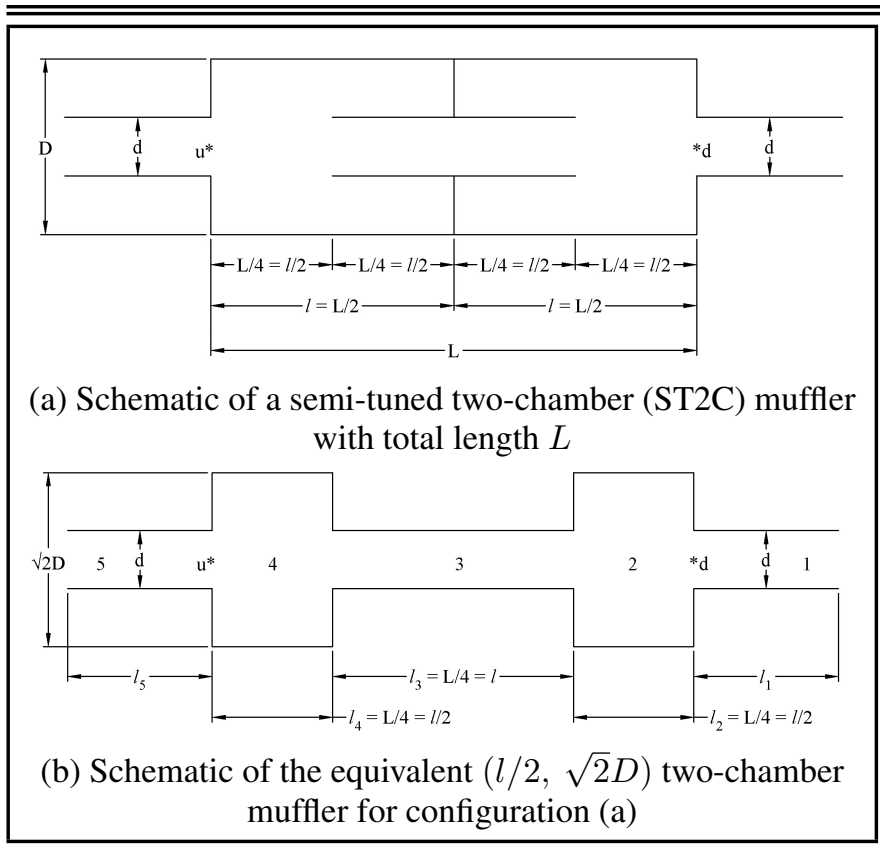

Figure 5. Analysis of an ST2C muffler using the equivalent configuration concept

Noting that TL is related to the velocity ratio $V R_{5}$ by Eq. (3), it can readily be seen that TL would peak at:

$$
\theta \equiv \frac{k L}{4}=\frac{\pi}{3} \text { and } \frac{2 \pi}{3}
$$

and drop in a sharp trough in between at:

$$
\frac{k L}{4}=\frac{\pi}{2} .
$$

Let us adopt dimensions of the muffler 19 of Fig. 11 of Davis et al., ${ }^{20}$ i.e., $L=48 "=1.1292 \mathrm{~m}, d=3 "=$ $0.0762 \mathrm{~m}, D=4 d=12 "=0.3048 \mathrm{~m}, c_{0}=1140 \mathrm{\prime} / \mathrm{s}=$ $347.5 \mathrm{~m} / \mathrm{s}$. Thus, area ratio:

$$
m=(D / d)^{2}=16
$$

Substituting these dimensions and data, it can be seen from Eq. (16) that the TL peaks would occur at:

$$
\begin{aligned}
& f_{p}=\frac{2 c_{0}}{3 L} \text { and; } \frac{4 c_{0}}{3 L} \\
& =\frac{2 \times 347.5}{3 \times 1.2192} \text { and } ; \frac{4 \times 347.5}{3 \times 1.2192} \\
& =190 \mathrm{~Hz} \text { and; } 380 \mathrm{~Hz} \text {; }
\end{aligned}
$$

and as per Eq. (17) the TL trough would occur at:

$$
f_{t}=\frac{c_{0}}{L}=\frac{347.5}{1.2192}=285 \mathrm{~Hz} \text {. }
$$

Use of Eq. (15) and Eq. (11) yields an estimate of TL at the peaks:

$$
\begin{aligned}
T L_{\text {peak }} \approx 20 \log \frac{\left|V R_{5 \text { peak }}\right|}{2} & =20 \log \frac{(2 \times 16)^{2}}{2}-3.75 \\
& \simeq 54.2-3.8=50.4 \mathrm{~dB}
\end{aligned}
$$

These deductions from the single most significant term in Velocity Ratio are borne out by Fig. 6(a), which is drawn

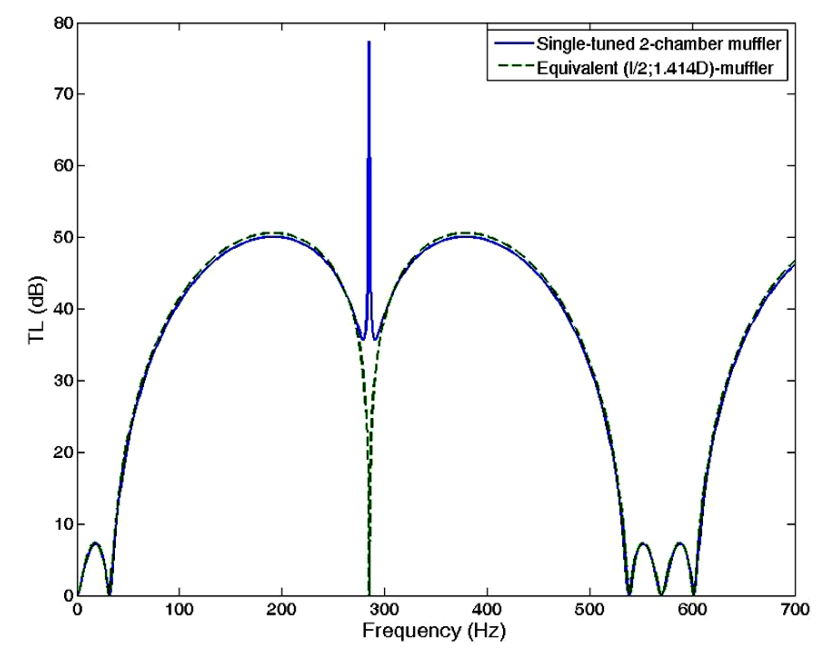

(a) TL comparison of the ST2C muffler and its equivalent $(l / 2,1.414 D)$ muffler

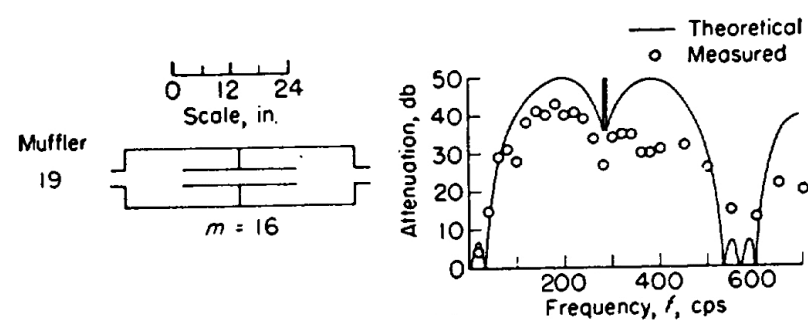

(b) Reproduction of Fig.11(d) from p.13 of the NACA 1192 report $^{20}$

Figure 6. Predicted and measured TL spectra of the semi-tuned two-chamber (ST2C) mufflers of Fig. 5(a) and Fig. 5(b)

from the proper transfer matrix multiplication, thereby reaffirming the utility as well as simplicity of the author's VRalgorithm. 1,17

For experimental validation of our predictions from the VR-algorithm, the graphs of Fig. 6(a) may be compared with the theoretical and experimental data adopted from the NACA report of Davis et al. ${ }^{20}-$ see Fig. 6(b).

The theoretical curves of Fig. 6(a) and Fig. 6(b) appear to match very well. However, comparison with measured values is not so good. One plausible explanation for this may be that the end corrections have not been subtracted from $L / 2$ while sizing the extended inlet and outlet of the intermediate pipe in muffler number 19 of the NACA Investigation. ${ }^{20}$ Significantly, however, the theoretical 3-D predictions match very well right up to $3000 \mathrm{~Hz}$ with the measured values of TL in Fig. 9 of Selamet et al.'s paper. ${ }^{15}$ This indicates that in the DT2C (or dual chamber) mufflers, where the intermediate chamber lengths are small, the evanescent higher-order modes may tunnel through, thereby necessitating a 3-D (FEM or analytical) analysis. This may, however, not be necessary from the practical point of view; one may as well design DT2C mufflers using plane-wave analysis coupled with the Equivalence principle and VelocityRatio algorithm, as illustrated here.

\section{TWO-DOUBLE TUNED SISO CHAMBERS IN SERIES}

All the tuned two-chamber mufflers considered above had co-axial inlet and outlet pipes. However, large diesel-generator (DG) sets have their exhaust mufflers lying horizontally on 


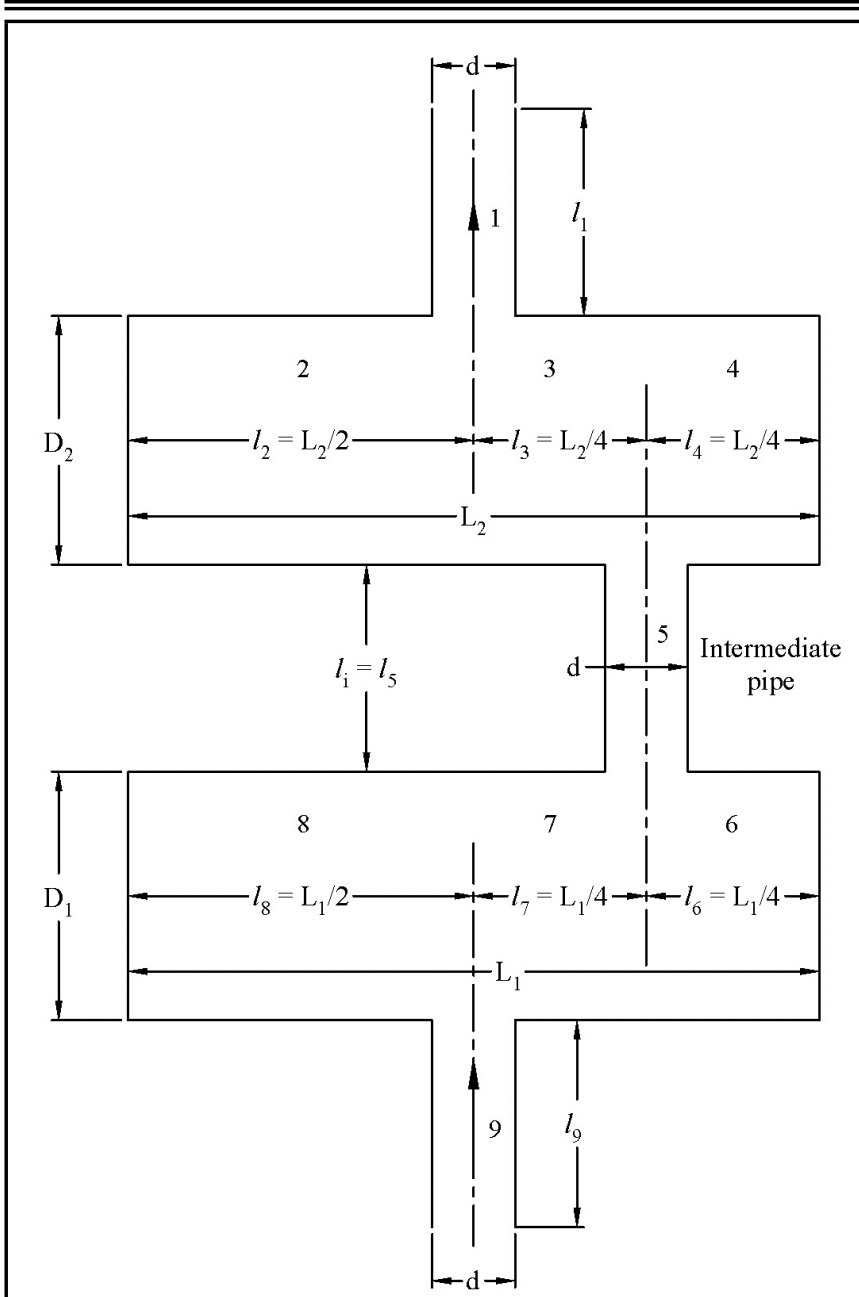

(a) Schematic of a double-tuned SISO two-chamber muffler

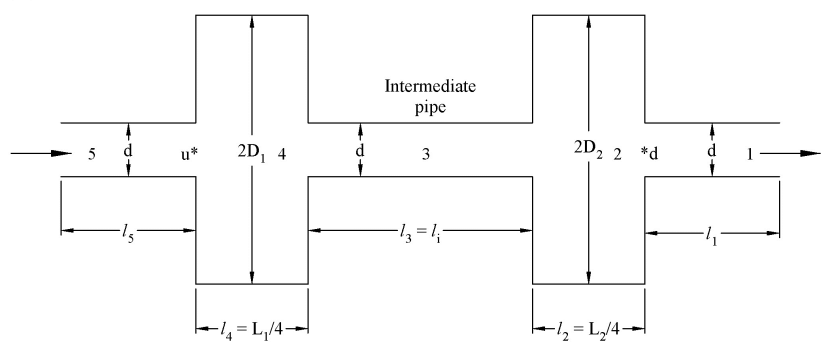

(b) Schematic of the equivalent $(L / 4,2 D)$ configuration for the muffler (a) above

Figure 7. Evaluation of TL of a double-tuned SISO two-chamber muffler using the equivalent configuration concept

the top of their acoustic enclosure or canopy, with SISO. A schematic of two double-tuned SISO chambers in series is shown in Fig. 7(a). Using the equivalence concept illustrated above in section 2, for practical purposes the muffler configuration of Fig. 7(a) can be represented by the equivalent configuration of Fig. 7(b).

This figure being formally similar to Fig. 3(b), its electrical analogous circuit should be the same as Fig. 3(c) and the one modified for anechoic source and load (terminations) should be the same as Fig. 3(d). Therefore, Eq. (2) to Eq. (7) should apply to the SISO configuration of Fig. 7 as well.

Further, if:

$$
L_{1}=L_{2}=L / 2 \text { and } D_{1}=D_{2}=D \text { and } D / d=3 ;
$$

so that the total muffler volume as well as the overall dimensions of Fig. 7 is the same as for the muffler of Fig. 3(a), then

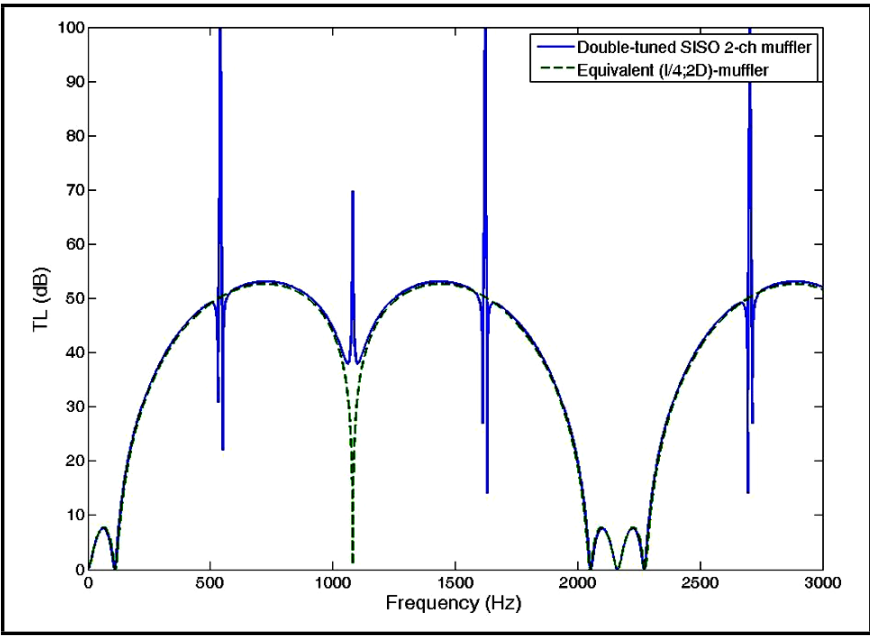

Figure 8. TL comparison of the DT-SISO-2C muffler and its practical equivalent $(l / 4 ; 2 D)$ muffler

Eq. (7) to Eq. (12) would apply to the muffler of Fig. 7(a) as well. This is borne out by Fig. 8 where the TL spectrum of the muffler configurations of Fig. 7(b) is compared with that of Fig. 7(a). As would be expected, the dashed curve of Fig. 8 is identical to the solid line curve of Fig. 4.

A scrutiny of the two curves in Fig. 8 again confirms the applicability of the concept of Equivalence, ${ }^{5,21}$ which simplifies the analysis and, in combination with the author's VRalgorithm, ${ }^{1,16,17}$ proves to be a boon for synthesis of the tunedchamber mufflers for wide-band transmission loss.

The low-frequency dip in Fig. 8 occurs at about $100 \mathrm{~Hz}$ which would be lower than the firing frequency of the typical multi-cylinder high-speed spark ignition engines. Additionally, the A-weighting correction at these frequencies would be of the order of $(-) 20 \mathrm{~dB}$. Of course, as pointed out below in section 5 , what matters in practice is insertion loss, IL. The wide-band character of the TL curves in Fig. 4, Fig. 6 and Fig. 8 should be reflected in the IL spectra as well.

\section{CONCLUDING REMARKS}

The concept of the equivalent $(L / 4,2 D)$ chamber to represent a double-tuned extended tube expansion chamber reduces the number of the constituent elements to nearly half as many.

Use of the Author's classical VR-algorithm along with electro-acoustic analogies eliminates the need to have to formulate and multiply-out a number of transfer matrices. Moreover, it enables us to directly identify the single most significant term that helps in predicting the salient features of the TL spectrum.

For the overall muffler dimensions considered in this paper, it is desirable to select a two-chamber configuration, with each chamber being double-tuned as shown in Figs. 3(a) and Fig. 7(a).

The TL-spectrum of the singly-tuned (or semi-tuned) twochamber muffler configuration of Fig. 5(a) has twice the number of troughs $(n=2,4,6,8,10 \ldots)$ compared to Fig. 3(a) $(n=4,8,12 \ldots)$. Besides, its domes are only $6 \mathrm{~dB}$ higher than those of the simple expansion chamber, as compared to $12 \mathrm{~dB}$ for the doubletuned configuration of Fig. 3(a).

The double-tuned SISO two-chamber muffler configuration of Fig. 7(a) has more or less identical TL-spectrum to that of the corresponding coaxial configuration of Fig. 3(a) for similar dimensions, as is borne out by the unbroken line curves of Fig. 4 and Fig. 8. 
While designing and fabricating, however, the following points must be kept in mind:

The equivalent configuration of Fig. 3(b) is hypothetical, where higher-order modes would start propagating at much lower frequencies due to the presence of short-length largediameter expansion chambers. It is the original configuration (Fig. 3(a)) that needs to be designed and prototyped.

Dimensions given in Fig. 3(a) are acoustical. For arriving at the physical (or geometrical) lengths we must subtract the end corrections ${ }^{4}$ from each of the extended lengths $L / 2$ and $L / 4$.

This paper presumes stationary inviscid medium. For waves in compressible mean flow, one needs to provide highly perforated bridges across the extended inlet/outlet. In that case, we have to subtract differential length as well as the modified end correction $^{22}$ from each of the acoustical lengths $L / 2$ and $L / 4$ in Fig. 3(a).

All discussion in this paper so far has been with respect to transmission loss (TL). In real practice, however, we have to design for insertion loss (IL) which represents the real reduction in noise due to the muffler. The IL curve is similar to the TL curve but has additional troughs due to the system resonance (the low-frequency dip) and tailpipe resonances. These aspects have been discussed in detail in Chapter 8 of the author's monograph. ${ }^{1}$

\section{ACKNOWLEDGEMENTS}

The author would like to acknowledge financial support of the Indian National Science Academy (INSA) through the grant of the INSA Senior Scientist position to him for the last five years, and now the grant of the INSA Honorary Scientist position for the next four years.

\section{REFERENCES}

1 Munjal, M. L. Acoustics of Ducts and Mufflers - Second Edition, Wiley, Chichester, UK, (2014).

2 Munjal, M. L., Galaitsis, A. G. and Ver, I. L. Passive Silencers, Noise and Vibration Control Engineering, Istvan L. Ver, Leo L. Beranek Eds., John Wiley, New York, (2006), 9.

3 Munjal, M. L. and Gowri, S. Theory and design of tuned extended tube chambers and concentric tube resonators, $J$. Acoust. Soc. India, 36(2), 53-71, (2009). https://dx.doi.org/

4 Chaitanya, P. and Munjal, M. L. Effect of wall thickness on the end corrections of the extended inlet and outlet of a double-tuned expansion chamber, Applied Acoustics, 72(1), 65-70, (2011). https://dx.doi.org/10.1016/j.apacoust.2010.09.001

5 Gaonkar, C. D. and Munjal, M. L. Theory of the double-tuned side-inlet side-outlet muffler, Noise Control Eng. J., 66(6), 489-495, (2018). https://dx.doi.org/10.3397/1/376641

6 Åbom, M. Derivation of four-pole parameters including higher order mode effects for expansion chamber mufflers with extended inlet and outlet, J. Sound Vib., 137 (3), 403-18, (1990). https://dx.doi.org/10.1016/0022460X(90)90807-C

7 Peat, K. S. The acoustical impedance at the junction of an extended inlet or outlet duct, J. Sound Vib., 150, 101-10, (1991). https://dx.doi.org/10.1016/0022-460X(91)90404-8
8 Selamet, A. and Ji, Z. L. Acoustical attenuation performance of circular expansion chambers with extended inlet/outlet, J. Sound Vib., 223(2), 197-212, (1999). https://dx.doi.org/10.1006/jsvi.1998.2138

9 Selamet, A. and Ji, Z. L. Acoustical attenuation performance of circular expansion chambers with offset inlet/outlet: I. Analytical approach, J. Sound Vib., 213(4), 601-17, (1998). https://dx.doi.org/10.1006/jsvi.1998.1514

10 Selamet, A. and Ji, Z. L. Acoustical attenuation performance of circular expansion chambers with offset inlet/outlet: II. Comparison with experimental and computational studies, J. Sound Vib., 213(4), 601-17,(1998). https://dx.doi.org/10.1006/jsvi.1998.1515

11 Torregrosa, A. J. Broatch, A., Payri, R. and Gonzalez, F., Numerical estimation of end corrections in extended-duct and perforated-duct mufflers, J. Vib. Acoust., 121(3), 302308, (1999). https://dx.doi.org/10.1115/1.2893980

12 Kang, Z. and Ji, Z. Acoustic length correction of duct extension into a cylindrical chamber, J. Sound Vib., 310, 782-91, (2008). https://dx.doi.org/10.1016/j.jsv.2007.11.005

13 Mimani, A. and Munjal, M. L. On the role of higher-order evanescent modes in end offset inlet and end-centered outlet elliptical flow-reversal chamber mufflers, Inter. J. Acoust. Vib., 17(3), 139-54, (2012). https://dx.doi.org/

14 Gaonkar, C. D., Rao, D. R., Kumar, K. M. and Munjal, M. L. End corrections for double-tuning of the sameend inlet-outlet muffler, Applied Acoustics, 159, (2020). https://dx.doi.org/10.1016/j.apacoust.2019.107116

15 Selamet, A., Denia, F. D., and Besa, A. J. Acoustic behaviour of circular dualchamber muffler, J. Sound Vib., 265, 967-985, (2003). https://dx.doi.org/10.1016/S0022$460 \mathrm{X}(02) 01258-0$

16 Munjal, M. L., Sreenath, A. V. and Narasimhan, M. V. Velocity ratio in analysis of linear dynamical systems, J. Sound Vib., 26(2), 173-191, (1973). https://dx.doi.org/10.1016/S0022-460X(73)80230-5

17 Munjal, M. L., Sreenath, A. V. and Narasimhan, M. V. An algebraic algorithm for the design and analysis of linear dynamical systems, J. Sound Vib., 26(2), 193-208, (1973). https://dx.doi.org/10.1016/S0022-460X(73)80231-7

18 Munjal, M. L. Narasimhan, M. V. and Sreenath, A. V. A rational approach to the synthesis of one-dimensional acoustic filters, J. Sound Vib., 29(3), 263-280, (1973). https://dx.doi.org/10.1016/S0022-460X(73)80284-6

19 Munjal, M. L. A rational synthesis of vibration isolators, J. Sound Vib., 39(2), 247-265, (1975). https://dx.doi.org/10.1016/S0022-460X(75)80223-9

20 Davis Jr., D. D., Stokes, M., Moore, D. and Stevens, L. NACA report 1192, Theoretical and experimental investigation of mufflers with comments on engine exhaust muffler design, (1954).

21 Gaonkar, C. D. and Munjal, M. L. Theory of the tuned sideinlet axial-outlet muffler, J. Acoust. Soc. India, 45(4), 195200, (2008).

22 Ramya, E. and Munjal, M. L. Improved tuning of the extended concentric tube resonator for wide-band transmission loss, Noise Control Eng. J., 62(4), 252-263, (2014). https://dx.doi.org/10.3397/1/376225 